\title{
Guaranteed Cost Fault-Tolerant Control for Networked Control Systems with Sensor Faults
}

\author{
Qixin Zhu, ${ }^{1,2}$ Kaihong $\mathrm{Lu}^{2}{ }^{2}$ Guangming Xie, ${ }^{3}$ and Yonghong $\mathrm{Zhu}^{4}$ \\ ${ }^{1}$ School of Mechanical Engineering, Suzhou University of Science and Technology, Suzhou 215009, China \\ ${ }^{2}$ School of Electronical and Electronic Engineering, East China Jiaotong University, Nanchang 330013, China \\ ${ }^{3}$ Department of Mechanics and Engineering Science, Peking University, Beijing 100871, China \\ ${ }^{4}$ School of Mechanical and Electronic Engineering, Jingdezhen Ceramic Institute, Jingdezhen 333001, China
}

Correspondence should be addressed to Qixin Zhu; bob21cn@163.com

Received 7 June 2014; Accepted 17 November 2014

Academic Editor: Jinhui Zhang

Copyright (C) 2015 Qixin Zhu et al. This is an open access article distributed under the Creative Commons Attribution License, which permits unrestricted use, distribution, and reproduction in any medium, provided the original work is properly cited.

\begin{abstract}
For the large scale and complicated structure of networked control systems, time-varying sensor faults could inevitably occur when the system works in a poor environment. Guaranteed cost fault-tolerant controller for the new networked control systems with time-varying sensor faults is designed in this paper. Based on time delay of the network transmission environment, the networked control systems with sensor faults are modeled as a discrete-time system with uncertain parameters. And the model of networked control systems is related to the boundary values of the sensor faults. Moreover, using Lyapunov stability theory and linear matrix inequalities (LMI) approach, the guaranteed cost fault-tolerant controller is verified to render such networked control systems asymptotically stable. Finally, simulations are included to demonstrate the theoretical results.
\end{abstract}

\section{Introduction}

Feedback control systems wherein the control loops are closed through a real-time network are called networked control systems (NCS) [1]. Due to their suitable and flexible structure, NCS is frequently encountered in practice for such fields as information technology, life science, and aeronautical and space technologies. However, there exist not only induced delay, data packet loss, and sequence disordering in NCS, but also actuators or sensors faults, which could cause negative impact on the performance of the system and even lead to system instability. Recently, the fault-tolerant control of NCS has become a new popular issue in the control field [2-9].

A fault-tolerant control algorithm for networked control systems is proposed based on Lyapunov stability theorem by Zheng and Fang [2]. Qu et al. have devised a faulttolerant robust control for a class of nonlinear uncertain systems with possible sensor faults considered and developed a robust measure to identify the stability- and performancevulnerable failures [3]. The faults of each sensor or actuator were taken as occurring randomly by Tian et al. [5], and their failure rates are governed by two sets of unrelated random variables satisfying certain probabilistic distribution. A sufficient condition is given by Zhang et al. [7], which could guarantee the stability of NCS with sensor failures or actuator failures and guarantee robustness to parameter uncertainties, but the issue of guaranteed cost is not discussed. A robust fault-tolerant control based on the integrity control theory when actuator faults occur is discussed by Zhang et al. [9]. Wang et al. [10] investigated the issue of integrity against actuator faults for NCS under variable-period sampling, in which the existence conditions of guaranteed cost faultstolerant control law are testified in terms of Lyapunov stability theory, but not referring to the effects of uncertain parameters.

Almost all literatures above consider the faults in some special cases. However, in practical application, because of large scale and complicated structure of NCS, the faults could vary from time to time when the system works in a poor environment. It is of great importance to explore a reasonable control method to guarantee the performance of NCS when 


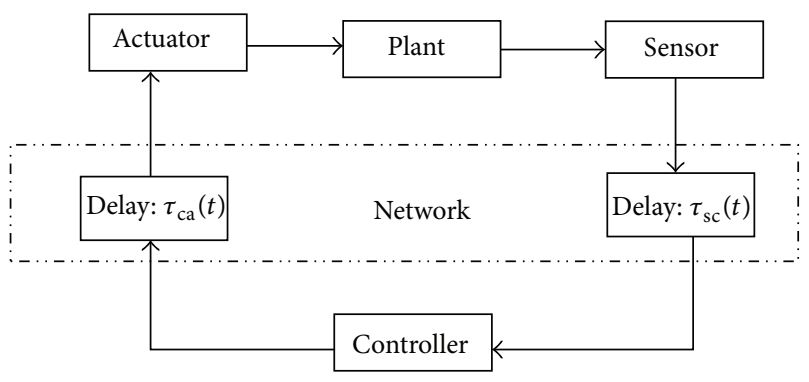

FIGURE 1: The structure of networked control systems.

time-varying faults occur. This motivates us to conduct the research work.

Based on the network transmission environment and sampling theory, the networked control systems are firstly modeled as a discrete-time closed-loop system by considering the time-varying transmission delay and sensor faults simultaneously. The model of NCS is related to the boundary values of the sensor faults. Using Lyapunov stability theory, a sufficient condition is given, which can render the closedloop NCS asymptotically stable and can guarantee it to meet the requirements of performance indicator (the upper bound of cost function). Based on LMI, the method of designing guaranteed cost fault-tolerant controller of NCS with timevarying faults is proposed in this paper.

\section{Modeling of Networked Control Systems with Sensor Faults}

A typical structure of NCS is shown in Figure 1.

In Figure 1, $\tau_{\mathrm{sc}}$ represents the transmission delay from sensor to the controller, while $\tau_{\mathrm{ca}}$ represents that from controller to the actuator. When the controller is static, the induced-delay time of system can be lumped as $\tau=\tau_{\mathrm{sc}}+\tau_{\mathrm{ca}}$.

A linear control plant is described by state equation as follows:

$$
\begin{aligned}
& \dot{x}(t)=A_{o} x(t)+B_{o} u(t) \\
& y(t)=C_{o} x(t)+D_{o} u(t),
\end{aligned}
$$

where $x \in R^{n}, u \in R^{m}$, and $y \in R^{r}$ represent state, input, and output vectors separately, while $A_{o}, B_{o}, C_{o}$, and $D_{o}$ are matrices with appropriate dimensions.

In order to facilitate the model, some rational assumptions for networked control systems are introduced as follows.

(A1) Single data package is transmitted. The packet loss and sequence disorder are not taken into consideration during the transmission process.

(A2) Uncertain time delay exists during the data transmission process. But time delay is bounded, and the maximum time delay does not exceed one sampling period; namely, $\tau \in[0, T]$, where $T$ is the sampling period.

(A3) Sensor is clock driving; controller and actuator are all event driving.
Based on the above assumptions (see Figure 2), within a sampling period, the system input is not a constant value but is a piecewise constant. In a cycle, system input can be described as

$$
u(t)= \begin{cases}u(k-1), & t_{k}<t \leq t_{k}+\tau_{k} \\ u(k), & t_{k}+\tau_{k}<t \leq t_{k}+T\end{cases}
$$

where $t_{k}$ is the $k$ th cycle sampling time and $\tau_{k}$ is the $k$ th cycle delay. follows:

The discrete-time model of system (1) can be obtained as

$$
\begin{aligned}
x(k+1) & =A x(k)+B_{1}\left(\tau_{k}\right) u(k)+B_{2}\left(\tau_{k}\right) u(k-1) \\
& =A x(k)+B_{1}\left(\tau_{k}\right) u(k)+\left(B-B_{1}\left(\tau_{k}\right)\right) u(k-1),
\end{aligned}
$$

where $A=e^{A_{o} T}, B_{1}\left(\tau_{k}\right)=\int_{0}^{T-\tau_{k}} e^{A_{o} t} B_{o} d t, B_{2}\left(\tau_{k}\right)=$ $\int_{T-\tau_{k}}^{T} e^{A_{o} t} B_{o} d t$, and $B=\int_{0}^{T} e^{A_{o} t} B_{o} d t$.

Moreover, according to the Jordan canonical form, matrix $A_{0}$ can be described as follows:

$$
A_{0}=\Lambda \operatorname{diag}\left(0_{p_{1} \times p_{1}}, \widehat{J}_{p_{2} \times p_{2}}, \breve{P}_{p_{3} \times p_{3}}\right) \Lambda^{-1},
$$

where $0 \leq p_{i} \leq n(i=1,2,3), p_{1}+p_{2}+p_{3}=n ; \widehat{J}$ is Jordan block that is a diagonal matrix consisting of the different eigenvalues with a total of $p_{2}$, denoted by $\lambda_{1}, \lambda_{2}, \ldots, \lambda_{p_{2}}$, while $\breve{J}$ is Jordan block for the repeated eigenvalues with a total of $p_{3}$, denoted by $\lambda_{0}$; matrix $\Lambda$ is the transformational matrix of Jordan canonical form for matrix $A_{0}$.

Correspondingly, the matrix $B_{1}$ with the variable $\tau_{k}$ can be equivalently calculated as

$$
\begin{array}{r}
B_{1}\left(\tau_{k}\right)=\Lambda \operatorname{diag}\left(T_{s}-\tau_{k}, \ldots, T_{s}-\tau_{k}, \frac{e^{\lambda_{1}\left(T_{s}-\tau_{k}\right)}-1}{\lambda_{1}},\right. \\
\left.\ldots, \frac{e^{\lambda_{p_{2}}\left(T_{s}-\tau_{k}\right)}-1}{\lambda_{p_{2}}}, J\right) \Lambda^{-1} B_{0},
\end{array}
$$

where

$$
J=\left[\begin{array}{cccc}
\frac{e^{\lambda_{0}\left(T_{s}-\tau_{k}\right)}-1}{\lambda_{0}} & \int_{0}^{T_{s}-\tau_{k}} t e^{\lambda_{0}} d t & \cdots & \frac{\int_{0}^{T_{s}-\tau_{k}} t e^{\lambda_{0}} d t}{\left(p_{3}-1\right) !} \\
0 & \frac{e^{\lambda_{0}\left(T_{s}-\tau_{k}\right)}-1}{\lambda_{0}} & \cdots & \frac{\int_{0}^{T_{s}-\tau_{k}} t e^{\lambda_{0}} d t}{\left(p_{3}-2\right) !} \\
\vdots & \vdots & \ddots & \vdots \\
0 & 0 & \cdots & \frac{e^{\lambda_{0}\left(T_{s}-\tau_{k}\right)}-1}{\lambda_{0}}
\end{array}\right]_{p_{3} \times p_{3}}
$$

Here a set of real numbers which are not equal to zero are denoted by $\alpha, \alpha_{1}, \alpha_{2}, \ldots, \alpha_{p_{2}}, \alpha_{0}$. 


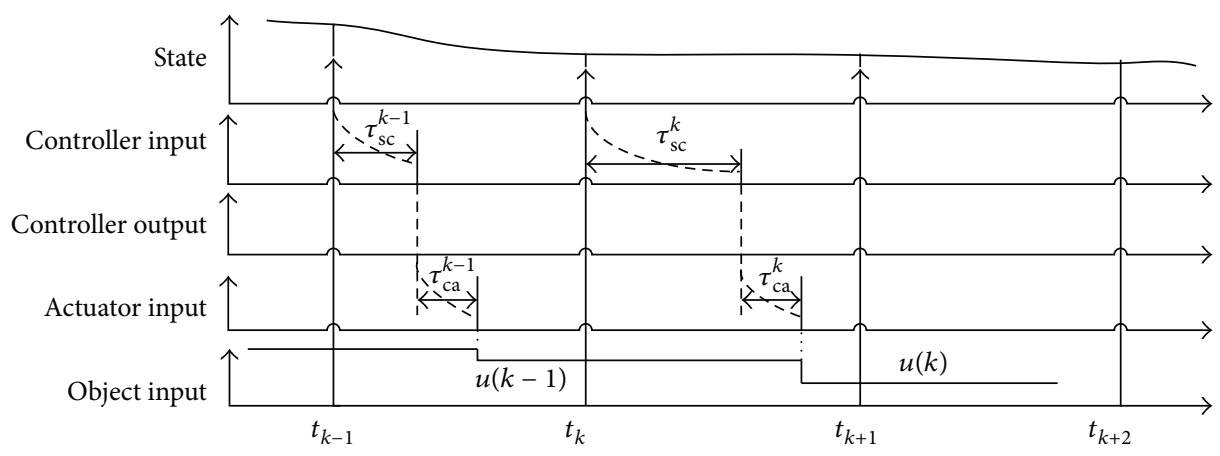

FIGURE 2: Timing diagram of signals transmitting in NCS.

And we define

$$
\begin{gathered}
E=\Lambda^{-1} B_{0} \\
F\left(\tau_{k}\right)=\operatorname{diag}\left(\frac{T_{s}-\tau_{k}}{\alpha}, \ldots, \frac{T_{s}-\tau_{k}}{\alpha}, \frac{e^{\lambda_{1}\left(T_{s}-\tau_{k}\right)}-1}{\alpha_{1} \lambda_{1}},\right. \\
\left.\ldots, \frac{e^{\lambda_{p_{2}}\left(T_{s}-\tau_{k}\right)}-1}{\alpha_{p_{2}} \lambda_{p_{2}}}, \frac{J}{\alpha_{0}}\right) .
\end{gathered}
$$

From (7), we know appropriate values of $\alpha, \alpha_{1}, \alpha_{2}, \ldots, \alpha_{p_{2}}, \alpha_{0}$ always can be chosen to satisfy $F^{T}\left(\tau_{k}\right) F\left(\tau_{k}\right) \leq I^{n \times n}$.

Comparing equality (7) with equality (5), we only need to define $D=\Lambda \operatorname{diag}\left(\alpha, \alpha_{1}, \alpha_{2}, \ldots, \alpha_{p_{2}}, \alpha_{0}\right)$; the matrix $B_{1}\left(\tau_{k}\right)$ can be expressed as

$$
B_{1}\left(\tau_{k}\right)=D F\left(\tau_{k}\right) E .
$$

Submitting equality (8) into equality (3), the following model can be obtained:

$$
\begin{aligned}
x(k+1)= & A x(k)+D F\left(\tau_{k}\right) E u(k) \\
& +\left(B-D F\left(\tau_{k}\right) E\right) u(k-1) .
\end{aligned}
$$

Assume that the system is fully measurable; state feedback is introduced as follows:

$$
u(k)=K x(k) \text {. }
$$

Considering the sensor faults that may occur, the controller is expanded as

$$
u^{F}(k)=K G(k) x(k) \text {, }
$$

where $u^{F}(k)=\left[u_{1}^{F}(k), u_{2}^{F}(k), \ldots, u_{m}^{F}(k)\right]^{T}$ represents faulty signal. $G(k)=\operatorname{diag}\left(g_{1}(k), g_{2}(k), \ldots, g_{m}(k)\right) ; g_{i}=0$ represents the fact that sensor $i$ faults occur; $g_{i}=1$ represents the fact that sensor $i$ is normal; $0 \leq g_{i} \leq 1$ represents the fact that partial faults occur at sensor $i$. When $G=I$, it represents the fact that all sensors are normal. The situation that all actuators' failure occurs at the same time is not taken into consideration here.

Moreover, the boundaries of faults usually can be measured in the practical work. The following definition is presented.
Definition 1. The upper bound of fault matrices is defined as follows:

$$
G_{u}=\operatorname{diag}\left(g_{u 1}, g_{u 2}, \ldots, g_{u n}\right), \quad 1 \geq g_{u i}>0,
$$

while the lower bound of fault matrices is defined as follows:

$$
G_{l}=\operatorname{diag}\left(g_{l 1}, g_{l 2}, \ldots, g_{l n}\right), \quad 1>g_{l i} \geq 0 .
$$

That is to say, $G(k) \in\left[G_{l}, G_{u}\right]$, which is time-varying. The mean value of matrices in Definition 1 can also be obtained as

$$
G_{0}=\operatorname{diag}\left(g_{01}, g_{02}, \ldots, g_{0 n}\right), \quad g_{0 i}=\frac{g_{u i}+g_{l i}}{2} ;
$$

furthermore, the following matrices are introduced:

$$
\begin{gathered}
L(k)=\operatorname{diag}\left(l_{1}(k), l_{2}(k), \ldots, l_{n}(k)\right), \\
l_{i}(k)=\frac{g_{i}(k)-g_{0 i}}{g_{0 i}} .
\end{gathered}
$$

Obviously, we have

$$
\begin{aligned}
-1 & \leq \frac{g_{l i}-g_{0 i}}{g_{0 i}} \leq l_{i}(k)=\frac{g_{i}(k)-g_{0 i}}{g_{0 i}} \\
& \leq \frac{g_{u i}-g_{0 i}}{g_{0 i}}=\frac{g_{u i}-g_{l i}}{g_{u i}+g_{l i}} \leq 1 .
\end{aligned}
$$

Based on (16), we have $-I_{n \times n} \leq L(k) \leq I_{n \times n}$. Based on (16), the following can be obtained:

$$
g_{i}=g_{0 i}\left(1+l_{i}\right), \quad i=1,2, \ldots n ;
$$

Naturally, we have $G=G_{0}(I+L)$.

The model of closed-loop systems with sensor faults can be obtained as

$$
\begin{aligned}
x(k+1)= & A x(k)+D F\left(\tau_{k}\right) E K G_{0}(I+L(k)) x(k) \\
& +\left(B-D F\left(\tau_{k}\right) E\right) K G_{0}(I+L(k-1)) x(k-1) .
\end{aligned}
$$

Remark 2. The NCS with time-varying delay and sensor faults is modeled as a closed-loop system (18) with the uncertain parameter $F\left(\tau_{k}\right)$ and time-varying parameter $L(k)$; unlike the previous models as [5], this model is related to the boundary values of the faults $G_{u}$ and $G_{l}$. Moreover, according 
to the expression of matrix $G_{0}$, we undoubtedly know it is invertible.

\section{The Design of Guaranteed Cost Fault-Tolerant Control}

For the system model (18) established above, the cost function is given as follows:

$$
\begin{aligned}
J_{\infty}=\sum_{k=0}^{\infty}\left\{x^{T}(k) Q x(k)+\left[K G_{0}(I+L) x(k)\right]^{T}\right. \\
\left.\times R K G_{0}(I+L) x(k)\right\}
\end{aligned}
$$

where $Q$ and $R$ are symmetric positive definite matrices.

To analyze the stability of the system expediently, the following lemmas are introduced.
Lemma 3 (Schur complement). For a symmetric matrix $S=$ $\left[\begin{array}{ll}S_{11} & S_{12} \\ S_{21} & S_{22}\end{array}\right]$, where $S_{11}=S_{11}^{T}, S_{12}^{T}=S_{21}$, and $S_{22}=S_{22}^{T}$, the following three conditions are equivalent:

(1) $S<0$;

(2) $S_{11}<0, S_{22}-S_{12}^{T} S_{11}^{-1} S_{12}<0$;

(3) $S_{22}<0, S_{11}-S_{12} S_{22}^{-1} S_{12}^{T}<0$.

Lemma 4 (see [11]). For any matrices $W, M, N$, or $F(t)$ with $F^{T} F \leq I$ and any scalar $\varepsilon>0$, the following inequality holds. $W+M F(t) N+N^{T} F^{T}(t) M^{T} \leq W+\varepsilon M M^{T}+\varepsilon^{-1} N^{T} N$.

Theorem 5. Given symmetric positive definite matrices $Q, R$ and gain matrix $K$, if symmetric positive definite matrices $P$ and $S$ exist, as well as a scalar $\varepsilon>0$, satisfying

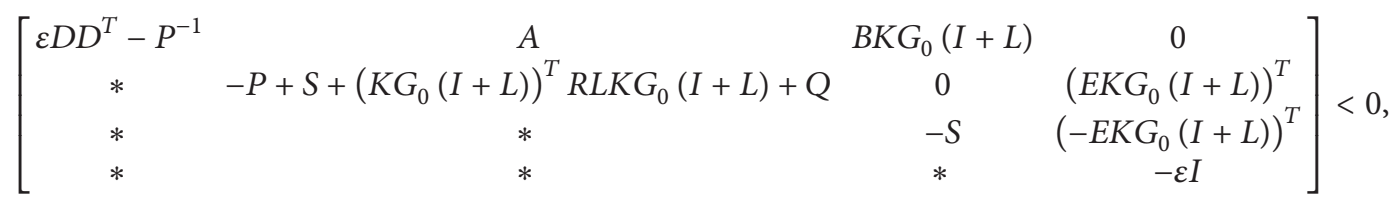

then the NCS (18) is asymptotically stable, where * represents the symmetry blocks of matrix.

Proof. Consider the following Lyapunov function:

$$
V(k)=x^{T}(k) P x(k)+x^{T}(k-1) S x(k-1) .
$$

For the convenience of writing, we denote $L=L(k)$ in the following expressions. Based on (18) conducting subtraction calculation can be obtained

$$
\begin{aligned}
\Delta V(k)= & v(k+1)-v(k) \\
= & x^{T}(k+1) P x(k+1)+x^{T}(k) S x(k) \\
& \quad-x^{T}(k) P x(k)-x^{T}(k-1) S x(k-1) \\
= & {\left[A x(k)+D F E K G_{0}(I+L) x(k)\right.} \\
& \left.\quad+(B-D F E) K G_{0}(I+L) x(k-1)\right]^{T} \\
& \quad \times \quad P\left[A x(k)+D F E K G_{0}(I+L) x(k)\right. \\
& \left.\quad+(B-D F E) K G_{0}(I+L) x(k-1)\right]
\end{aligned}
$$

$$
\begin{aligned}
& +x^{T}(k) S x(k)-x^{T}(k) P x(k) \\
& -x^{T}(k-1) S x(k-1) \\
= & {\left[\begin{array}{c}
x(k) \\
x(k-1)
\end{array}\right]^{T}\left[\begin{array}{cc}
\Gamma^{T} P \Gamma-P+S & \Gamma^{T} P \Omega \\
* & \Omega^{T} P \Omega-S
\end{array}\right] } \\
& \times\left[\begin{array}{c}
x(k) \\
x(k-1)
\end{array}\right],
\end{aligned}
$$

where $\Gamma=A+\operatorname{DFEKG}_{0}(I+L)$ and $\Omega=B K G_{0}(I+L)-$ $\operatorname{DFEKG}_{0}(I+L)$.

System (18) is asymptotically stable, only if it satisfies $\Delta V<0$. It is equivalent to

$$
\left[\begin{array}{cc}
\Gamma^{T} P \Gamma-P+S & \Gamma^{T} P \Omega \\
* & \Omega^{T} P \Omega-S
\end{array}\right]<0 .
$$

Now, take the following inequality into consideration:

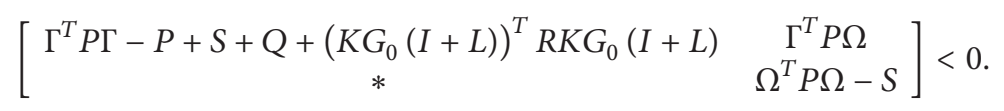


Inequality (24) is equivalent to

$$
\begin{aligned}
& {\left[\begin{array}{cc}
\Gamma^{T} P \Gamma-P+S & \Gamma^{T} P \Omega \\
* & \Omega^{T} P \Omega-S
\end{array}\right]}
\end{aligned}
$$

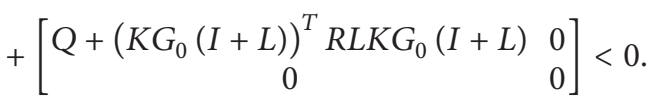

$Q$ and $R$ are symmetric positive definite matrices; therefore, inequality (24) is a sufficient condition of inequality (23). Inequality (24) can be expressed as follows:

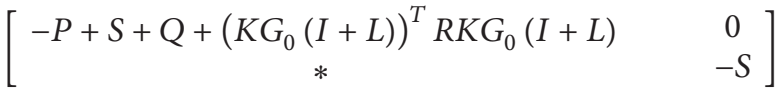

$$
\begin{aligned}
& +\left[\begin{array}{l}
\Gamma^{T} \\
\Omega^{T}
\end{array}\right] P\left[\begin{array}{ll}
\Gamma & \Omega
\end{array}\right]<0 .
\end{aligned}
$$

From Lemma 3, it follows that

$$
\begin{aligned}
& {\left[\begin{array}{ccc}
-P^{-1} & \Gamma & \Omega \\
* & -P+S+Q+\left(K G_{0}(I+L)\right)^{T} R K G_{0}(I+L) & 0 \\
* & * & -S
\end{array}\right]} \\
& <0 \text {. }
\end{aligned}
$$

Inequality (27) can be rewritten as

$$
\begin{aligned}
& {\left[\begin{array}{ccc}
-P^{-1} & A & B K G_{0}(I+L) \\
* & -P+S+Q+\left(K G_{0}(I+L)\right)^{T} R K G_{0}(I+L) & 0 \\
* & * & -S
\end{array}\right]+\left[\begin{array}{l}
D \\
0 \\
0
\end{array}\right] F\left[\begin{array}{lll}
0 & E K G_{0}(I+L) & -E K G_{0}(I+L)
\end{array}\right]} \\
& +\left[\begin{array}{lll}
0 & E K G_{0}(I+L) & -E K G_{0}(I+L)
\end{array}\right]^{T} F^{T}\left[\begin{array}{c}
D \\
0 \\
0
\end{array}\right]^{T}<0 .
\end{aligned}
$$

From Lemma 4, the sufficient condition of inequality (28) follows that

$$
\begin{aligned}
& {\left[\begin{array}{ccc}
-P^{-1} & A & B K G_{0}(I+L) \\
* & -P+S+Q+\left(K G_{0}(I+L)\right)^{T} R K G_{0}(I+L) & 0 \\
* & * & -S
\end{array}\right]} \\
& +\varepsilon^{-1}\left[\begin{array}{c}
0 \\
\left(E K G_{0}(I+L)\right)^{T} \\
\left(-E K G_{0}(I+L)\right)^{T}
\end{array}\right]\left[\begin{array}{c}
0 \\
\left(E K G_{0}(I+L)\right)^{T} \\
\left(-E K G_{0}(I+L)\right)^{T}
\end{array}\right]^{T}+\varepsilon\left[\begin{array}{c}
D \\
0 \\
0
\end{array}\right]\left[\begin{array}{lll}
D^{T} & 0 & 0
\end{array}\right]<0 .
\end{aligned}
$$

Therefore, inequality (29) is a sufficient condition of inequality (23). That is to say, if inequality (29) exists, NCS (18) is asymptotically stable. From Lemma 3 , the inequality above is equivalent to inequality (20). This completes the proof.

Theorem 6. If the conditions of Theorem 5 are satisfied, for all allowable uncertainties of system (18), its cost function is defined as (19) satisfying

$$
J_{\infty} \leq J_{0}=x^{T}(0) P x(0)+x^{T}(-1) S x(-1) .
$$

Proof. If inequality (20) exists, inequality (25) must exist.
Pre- and postmultiplying (25) by $\left[\begin{array}{c}x(k) \\ x(k-1)\end{array}\right]^{T}$ and by $\left[\begin{array}{c}x(k) \\ x(k-1)\end{array}\right]$ separately, it follows that

$$
\begin{aligned}
& {\left[\begin{array}{c}
x(k) \\
x(k-1)
\end{array}\right]^{T}\left[\begin{array}{cc}
\Gamma^{T} P \Gamma-P+S & \Gamma^{T} P \Omega \\
* & \Omega^{T} P \Omega-S
\end{array}\right]\left[\begin{array}{c}
x(k) \\
x(k-1)
\end{array}\right]} \\
& \leq\left[\begin{array}{c}
x(k) \\
x(k-1)
\end{array}\right]^{T}\left[\begin{array}{cc}
-Q-\left(K G_{0}(I+L)\right)^{T} R K G_{0}(I+L) & 0 \\
0 & 0
\end{array}\right] \\
& \quad \times\left[\begin{array}{c}
x(k) \\
x(k-1)
\end{array}\right] \\
& =x^{T}(k)\left(-Q-\left(K G_{0}(I+L)\right)^{T} R K G_{0}(I+L)\right) x(k) .
\end{aligned}
$$


Therefore,

$$
\begin{aligned}
J_{\infty} & \leq-\sum_{k=0}^{\infty} \Delta V(k)=V(0)-\lim _{k \rightarrow \infty} V(k) \\
& =x^{T}(0) P x(0)+x^{T}(-1) S x(-1)-\lim _{k \rightarrow \infty} V(k) .
\end{aligned}
$$

According to Theorem 5, system (18) is asymptotically stable. Therefore, $\lim _{k \rightarrow \infty} x(k)=\lim _{k \rightarrow \infty} x(k-1)=0$. Logically, there must exist $\lim _{k \rightarrow \infty} V(k)=0$. Inserting it into (32), inequality (30) can be obtained.

Theorem 7. Given symmetric positive definite matrices $Q$ and $R$, as well as a set of constants $\sigma_{1}$ and $\sigma_{2}$, if symmetric positive definite matrices $X, Y$ and matrix $W$ exist, as well as scalar $\varepsilon>0$, satisfying the following LMI:

$$
\left[\begin{array}{cccccccccc}
-Q^{-1} & 0 & 0 & 0 & 0 & 0 & 0 & X & 0 & 0 \\
* & -\sigma_{1} I & 0 & 0 & 0 & 0 & 0 & X & 0 & 0 \\
* & * & -\sigma_{2} I & 0 & 0 & 0 & 0 & 0 & X & 0 \\
* & * & * & -\sigma_{1} X & 0 & W^{T} & 0 & 0 & 0 & (E W)^{T} \\
* & * & * & * & -\sigma_{2} X & 0 & (B W)^{T} & 0 & 0 & (-E W)^{T} \\
* & * & * & * & * & -R^{-1} & 0 & W & 0 & 0 \\
* & * & * & * & * & * & \varepsilon D D^{T}-X & A & B W & 0 \\
* & * & * & * & * & * & * & -X+Y & 0 & (E W)^{T} \\
* & * & * & * & * & * & * & * & -Y & (-E W)^{T} \\
* & * & * & * & * & * & * & * & * & -\varepsilon I
\end{array}\right]<0
$$

then the NCS (18) is asymptotically stable with control gain $K=W X^{-1} G_{0}^{-1}$, and for all allowable uncertainties of system, its performance indicator defined as (19) satisfies inequality (30).
Proof. If inequality (20) exists, Theorems 5 and 6 must exist. Inequality (20) is equivalent to

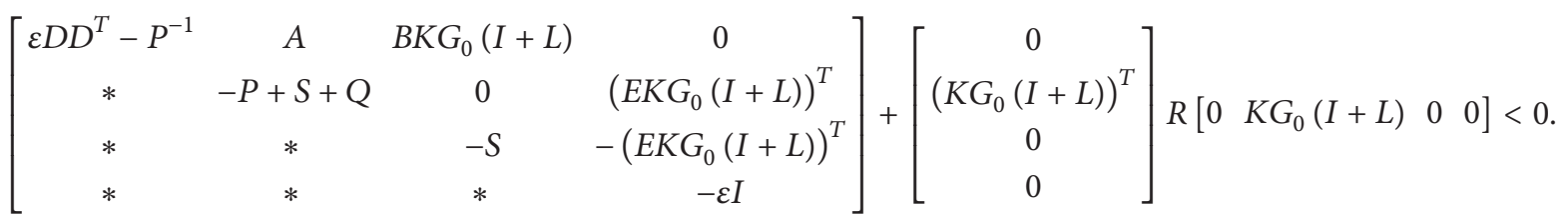

From Lemma 3, it follows that

$$
\left[\begin{array}{ccccc}
-R^{-1} & 0 & K G_{0}(I+L) & 0 & 0 \\
* & \varepsilon D D^{T}-P^{-1} & A & B K G_{0}(I+L) & 0 \\
* & * & -P+S+Q & 0 & \left(E K G_{0}(I+L)\right)^{T} \\
* & * & * & -P & \left(-E K G_{0}(I+L)\right)^{T} \\
* & * & * & * & -\varepsilon I
\end{array}\right]<0
$$


Inequality (35) can be rewritten as

where

$$
\Phi+\Xi_{1} L \widehat{I}_{1}+\Xi_{2} L \widehat{I}_{2}+\left(\Xi_{1} L \widehat{I}_{1}\right)^{T}+\left(\Xi_{2} L \widehat{I}_{2}\right)^{T}<0,
$$

$$
\begin{gathered}
\Phi=\left[\begin{array}{ccccc}
-R^{-1} & 0 & K G_{0} & 0 & 0 \\
* & \varepsilon D D^{T}-P^{-1} & A & B K G_{0} & 0 \\
* & * & -P+S+Q & 0 & \left(E K G_{0}\right)^{T} \\
* & * & * & -P & \left(-E K G_{0}\right)^{T} \\
* & * & * & * & -\varepsilon I
\end{array}\right]<0, \\
\Xi_{1}=\left[\begin{array}{c}
K G_{0} \\
0 \\
0 \\
0 \\
E K G_{0}
\end{array}\right], \quad \widehat{I}_{1}=\left[\begin{array}{c}
0 \\
0 \\
I \\
0 \\
0
\end{array}\right], \quad \Xi_{2}=\left[\begin{array}{c}
0 \\
B K G_{0} \\
0 \\
0 \\
-E K G_{0}
\end{array}\right], \quad \widehat{I}_{1}=\left[\begin{array}{l}
0 \\
0 \\
0 \\
I \\
0
\end{array}\right]^{T} .
\end{gathered}
$$

Using Lemma 4, it can be obtained that

$$
\Phi+\sigma_{1}{ }^{-1} \Xi_{1} \Xi_{1}^{T}+\sigma_{1} \widehat{I}_{1}^{T} \widehat{I}_{1}+\sigma_{2}{ }^{-1} \Xi_{2} \Xi_{2}^{T}+\sigma_{2} \widehat{I}_{2}^{T} \widehat{I}_{2}<0 .
$$

Using Lemma 3 repeatedly, the following inequality can be obtained:

$$
\left[\begin{array}{cccccccccc}
-Q^{-1} & 0 & 0 & 0 & 0 & 0 & 0 & I & 0 & 0 \\
* & -\sigma_{1}{ }^{-1} I & 0 & 0 & 0 & 0 & 0 & I & 0 & 0 \\
* & * & -\sigma_{2}{ }^{-1} I & 0 & 0 & 0 & 0 & 0 & I & 0 \\
* & * & * & -\sigma_{1} I & 0 & \left(K G_{0}\right)^{T} & 0 & 0 & 0 & \left(E K G_{0}\right)^{T} \\
* & * & * & * & -\sigma_{2} I & 0 & \left(B K G_{0}\right)^{T} & 0 & 0 & \left(-E K G_{0}\right)^{T} \\
* & * & * & * & * & -R^{-1} & 0 & K G_{0} & 0 & 0 \\
* & * & * & * & * & * & \varepsilon D D^{T}-P^{-1} & A & B K G_{0} & 0 \\
* & * & * & * & * & * & * & -P+S & 0 & \left(E K G_{0}\right)^{T} \\
* & * & * & * & * & * & * & * & -S & \left(-E K G_{0}\right)^{T} \\
* & * & * & * & * & * & * & * & * & -\varepsilon I
\end{array}\right]<.
$$

Pre- and postmultiplying (39) by block-diag $\left(I, \sigma_{1} I, \sigma_{2} I, P^{-1}\right.$, $\left.P^{-1}, I, I, P^{-1}, P^{-1}, I\right)$ and then letting $X=P^{-1}, W=$ $K G_{0} P^{-1}=K G_{0} X, Y=P^{-1} S P^{-1}$, inequality (33) can be obtained. Because $G_{0}$ is invertible, $K$ can be obtained by calculating $K=W X^{-1} G_{0}^{-1}$. This completes the proof.

\section{Simulations}

Consider the model of inverted pendulum device as follows:

$$
\dot{x}(t)=\left[\begin{array}{cccc}
-0.5 & -0.51 & 0 & 0 \\
0 & -1.3 & 0 & 0 \\
0.1 & 0.2 & -0.3 & 0 \\
0 & 0 & 0 & 0.3
\end{array}\right] x(t)+\left[\begin{array}{c}
0.1 \\
1 \\
0.5 \\
1.2
\end{array}\right] u(t)
$$

Consider the sampling period $T=0.1 \mathrm{~s}$; the network induced time delay satisfies $\tau_{k}<T$ and is time-varying. We choose the parameters as $\alpha_{1}=1.3, \alpha_{2}=1.8, \alpha_{3}=-1.1$, and $\alpha_{4}=1.5$. Computing (5)-(8), we have

$$
\begin{gathered}
D=\left[\begin{array}{cccc}
0 & 1.61 & -0.5772 & 0 \\
0 & 0 & -0.9054 & 0 \\
1.3 & -0.805 & 0.2388 & 0 \\
0 & 0 & 0 & 0
\end{array}\right], \quad E=\left[\begin{array}{c}
0.495 \\
-0.6009 \\
1.214 \\
1.2
\end{array}\right. \\
F\left(\tau_{k}\right)=\operatorname{diag}\left(\frac{e^{-0.3\left(0.1-\tau_{k}\right)}-1}{-0.39}, \frac{e^{-0.5\left(0.1-\tau_{k}\right)}-1}{-0.9},\right. \\
\left.\frac{e^{-1.3\left(0.1-\tau_{k}\right)}-1}{1.43}, \frac{e^{0.3\left(0.1-\tau_{k}\right)}-1}{0.45}\right) .
\end{gathered}
$$

Obviously, $F^{T}\left(\tau_{k}\right) F\left(\tau_{k}\right)<I$ is satisfied. 
TABLE 1: The boundaries of sensor faults.

\begin{tabular}{lccc}
\hline Upper bound & Lower bound \\
\hline Sensor faults matrix $G$ & {$\left[\begin{array}{ccccc}0.9 & 0 & 0 & 0 \\
0 & 0.9 & 0 & 0 \\
0 & 0 & 0.86 & 0 \\
0 & 0 & 0 & 0.78\end{array}\right]$} \\
\hline
\end{tabular}

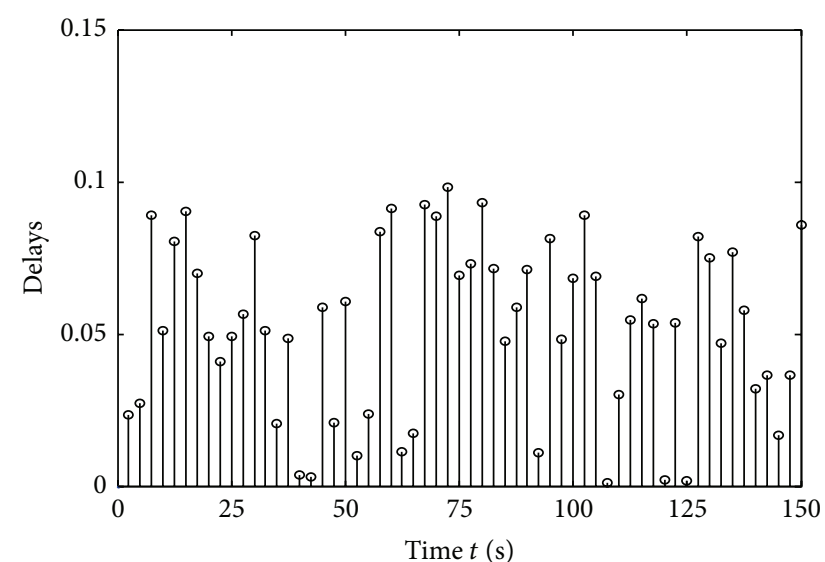

FIGURE 3: The distribution of delays in NCS.

And the following parameters are given:

$$
Q=\left[\begin{array}{cccc}
50 & 0 & 0 & 0 \\
0 & 80 & 0 & 0 \\
0 & 0 & 130 & 0 \\
0 & 0 & 0 & 220
\end{array}\right], \quad R=1800, \sigma_{1}=\sigma_{2}=10^{9}
$$

Considering the sensor faults that may occur, the boundaries of faults are given in Table 1.

By making use of LMI toolbox in MATLAB to solve the linear matrix inequality (33), guaranteed cost fault-tolerant controller parameters of NCS can be obtained:

$$
K=W X^{-1} G_{0}^{-1}=\left[\begin{array}{llll}
-0.0756 & -1.03 & -0.7531 & -1.9025
\end{array}\right] .
$$

It assumes the initial state of the system is $x(0)=x(-1)=$

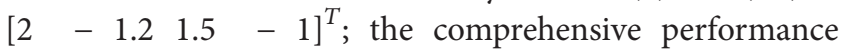
indicator can be obtained $J_{0}=16.3685$. The time-varying transmission delays produced in network are shown in Figure 3. When all sensors are normal, namely, $G=I$, the state responses of NCS are shown in Figure 4, from which we know the system gets steady at $40 \mathrm{~s}$. When sensor faults vary in the scope of boundary values in Table 1, the state responses of NCS are shown in Figure 5, from which we can see the transition time of state response obviously becomes longer than that in Figure 4 because of the effects of time-varying sensor faults, but the system is still asymptotically stable and gets steady at $80 \mathrm{~s}$. So, the performance of NCS can be well maintained by the guaranteed cost fault-tolerant controller, which demonstrates the effectiveness and feasibility of the approach proposed in this paper.

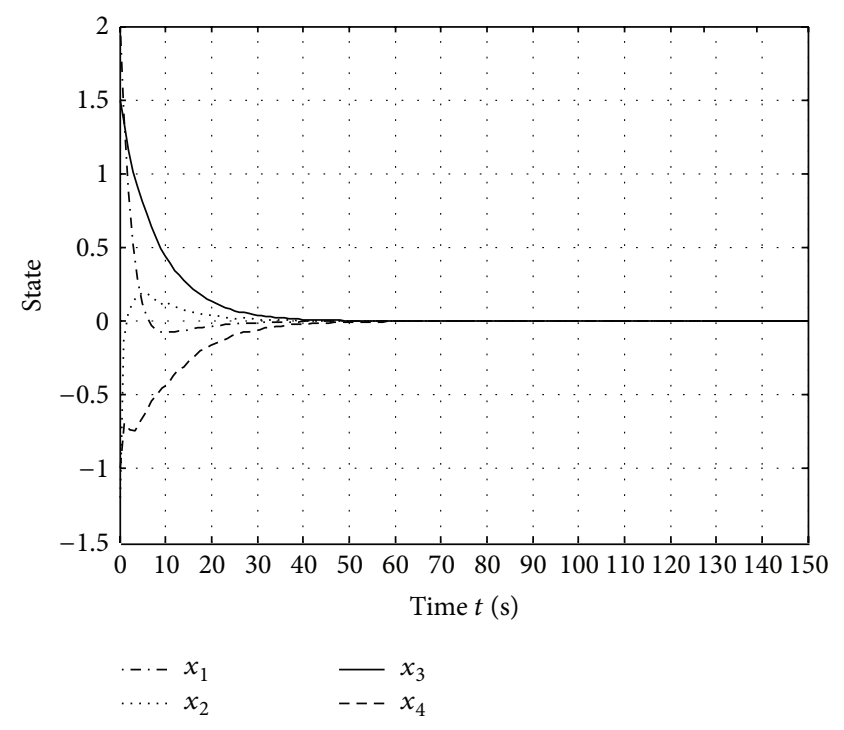

FIGURE 4: State response of NCS without sensor faults.

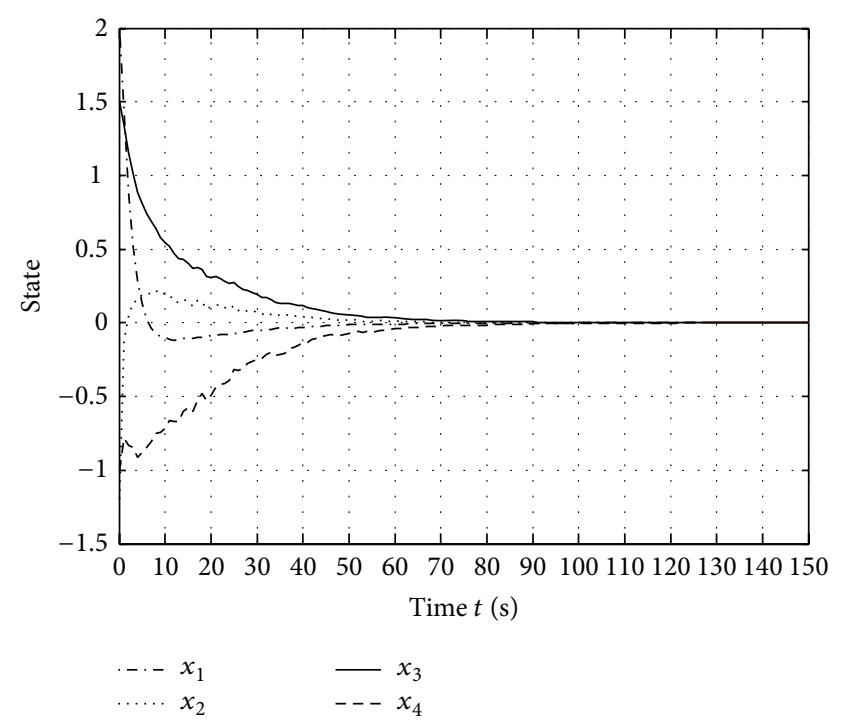

FIGURE 5: State response of NCS with time-varying sensor faults.

\section{Conclusions}

When time-varying sensor faults occur, guaranteed cost fault-tolerant control problem of networked control systems is studied in this paper. Using Lyapunov stability theory and linear matrix inequality (LMI) approach, a sufficient condition is given, which can render the closed-loop NCS 
with sensor faults asymptotically stable and can guarantee it to meet the requirements of performance indicator. And based on LMI, the method of designing guaranteed cost faulttolerant controller is proposed. Moreover, the feasibility and effectiveness of this method have been demonstrated by a simulation example. The next research task will be analyzing the time-varying actuator faults to improve the performance of NCS further.

\section{Conflict of Interests}

The authors declare that there is no conflict of interests regarding the publication of this paper.

\section{Acknowledgments}

This work was partly supported by the National Nature Science Foundation of China (61164014, 51375323) and Qing Lan Project of Jiangsu Province, China.

\section{References}

[1] J. K. Yook, D. M. Tilbury, and N. R. Soparkar, "Trading computation for bandwidth: reducing communication in distributed control systems using state estimators," IEEE Transactions on Control Systems Technology, vol. 10, no. 4, pp. 503-518, 2002.

[2] Y. Zheng and H. J. Fang, "Robust fault tolerant control of networked control system with time-varying delays," Journal of Xian Jiaotong University, vol. 38, no. 8, pp. 804-807, 2004.

[3] Z. Qu, C. M. Ihlefeld, Y. Jin, and A. Saengdeejing, "Robust faulttolerant self-recovering control of nonlinear uncertain systems," Automatica, vol. 39, no. 10, pp. 1763-1771, 2003.

[4] C. Peng, T. C. Yang, and E. G. Tian, "Robust fault-tolerant control of networked control systems with stochastic actuator failure," IET Control Theory \& Applications, vol. 4, no. 12, pp. 3003-3011, 2010.

[5] E. Tian, D. Yue, and C. Peng, "Brief Paper: reliable control for networked control systems with probabilistic sensors and actuators faults," IET Control Theory and Applications, vol. 4, no. 8, pp. 1478-1488, 2010.

[6] Y. Zhang and J. Jiang, "Bibliographical review on reconfigurable fault-tolerant control systems," Annual Reviews in Control, vol. 32, no. 2, pp. 229-252, 2008.

[7] J.-H. Zhang, J.-L. Li, and Y. Chen, "Robust fault tolerant control for a kind of uncertain networked control system," Journal of Kunming University of Science and Technology, vol. 37, no. 1, pp. 36-40, 2012 (Chinese).

[8] Q. Zhu, K. Lu, and Y. Zhu, "Robust guaranteed cost control for networked control system with actuator failure," International Journal of Applied Mathematics \& Statistics, vol. 50, no. 20, pp. 55-68, 2013.

[9] W. Zhang, M. S. Branicky, and S. M. Phillips, "Stability of networked control systems," IEEE Control Systems Magazine, vol. 21, no. 1, pp. 84-99, 2001.

[10] Y. Wang, L. Xie, and C. E. de Souza, "Robust control of a class of uncertain nonlinear systems," Systems \& Control Letters, vol. 19, no. 2, pp. 139-149, 1992.

[11] X. Y. Luo, M. J. Shang, C. L. Chen, and X. P. Guan, "Guaranteed cost active fault-tolerant control of networked control system with packet dropout and transmission delay," International Journal of Automation and Computing, vol. 7, no. 4, pp. 509-515, 2010. 


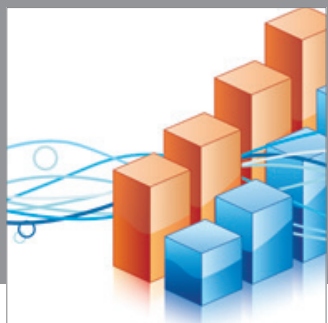

Advances in

Operations Research

mansans

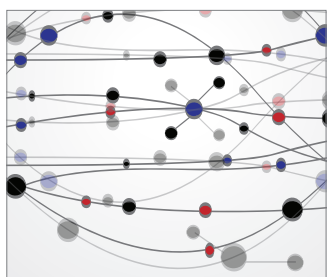

The Scientific World Journal
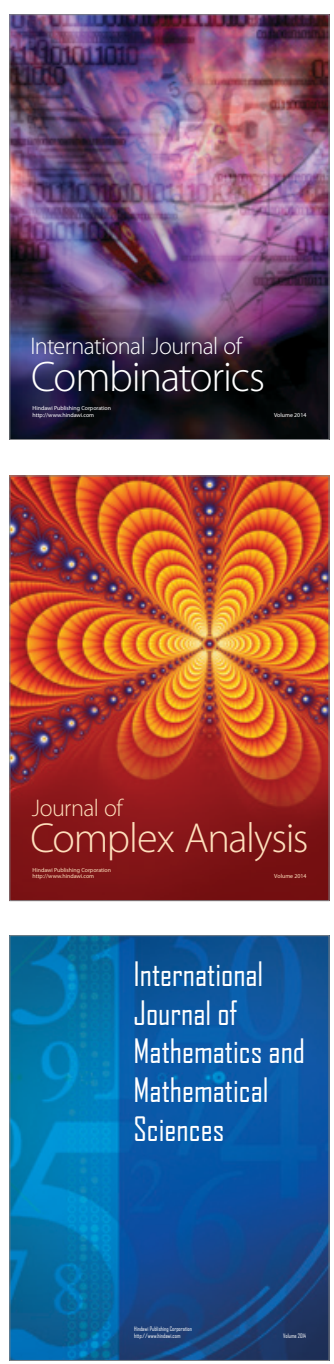
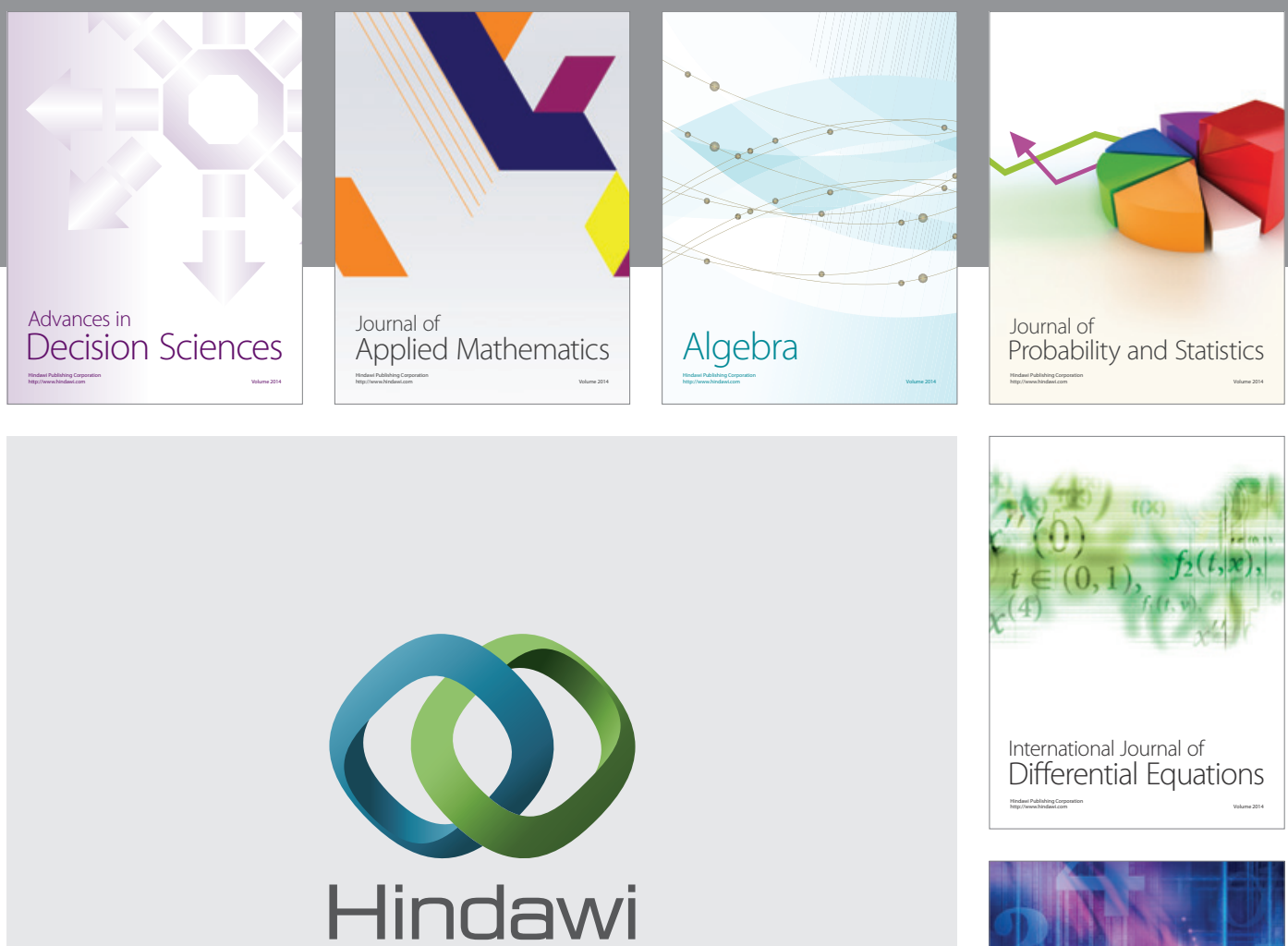

Submit your manuscripts at http://www.hindawi.com
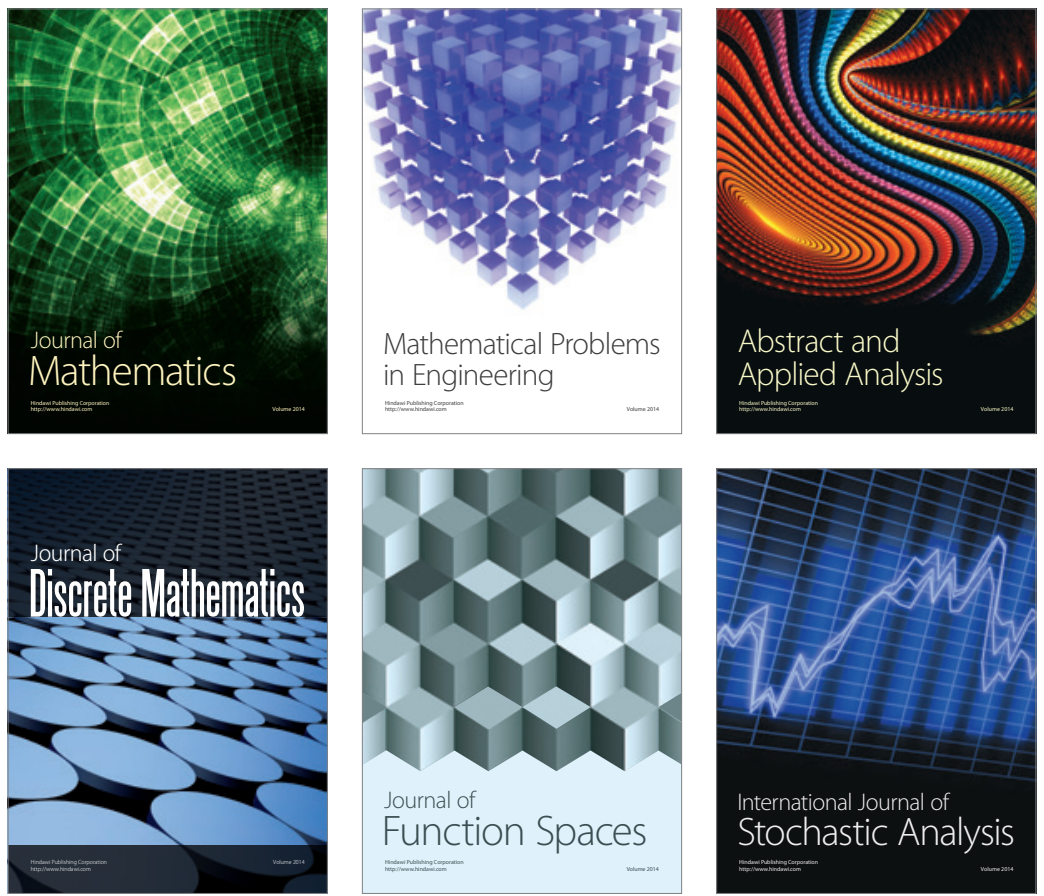

Journal of

Function Spaces

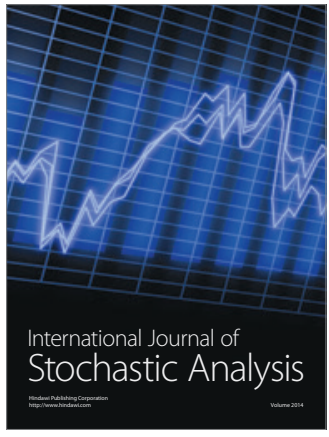

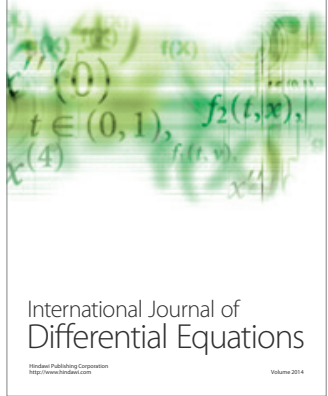
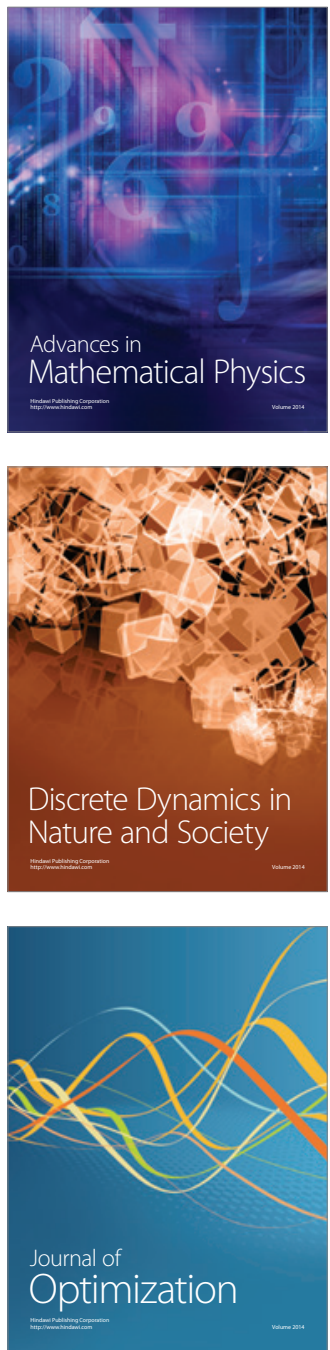